FUTURE LEPTON COLLIDERS AND LASER ACCELERATION

Zohreh Parsa

Brookhaven National Laboratory

Physics Department

Upton, New York

March 2000

Submitted to $3^{\text {rd }}$ International workshop on "Electron-Electron Interactions at TeV

Energies (e-e-99)", Univ. of Calif., Santa Cruz, Dec. 10-12, 1999. 


\title{
FUTURE LEPTON COLLIDERS AND LASER ACCELERATION
}

\author{
Zohreh Parsa \\ Brookhaven National Laboratory, \\ Physics Department 510A \\ Upton, New York 11973-5000, USA
}

E-mail: parsa@bnl.gov

\footnotetext{
* Supported by L.S. Department of Energy Cinder Contract. No. DE-AC02-98CH108s6.

- Submitied for the $3^{\text {rd }}$ Interaational Wiorkshop on "Electron - Electron Interactions at TeV Energies (e-e-99)", Lniversity of California, Santa Cruz, December 10-12, 1999. To be published in International Journal of Modera Physics A. World Scientific Publishing Company ( 2000$)$.
} 


\title{
FUTURE LEPTON COLLIDERS AND LASER ACCELERATION
}

\author{
ZOHREH PARSA * \\ Brookhaven National Laboratory \\ Upton, New York 11973-5000, USA
}

\begin{abstract}
Future high energy colliders along with their physics potential, and relationship to new laser technology are discussed. Experimental approaches and requirements for "New Physics" exploration are also described.
\end{abstract}

\section{Introduction}

Particle beam colliders are the primary tools for performing high energy physics research. Collisions of high energy particles produce events in which much of the energy of the beams can be converted into the masses of new heavy particles not normally found in nature. By studying the production and decay of these new particles, the underlying structure of the universe and the laws that govern it are unveiled.

In devising a strategy for future technical accelerator initiatives we can look at the historical record as represented by the Livingston Plot in Figure 1. There, the energy scale probed is plotted as function of calendar time for various accelerators. It shows where we come from, where we are and where we are going. ${ }^{1}$

Experiments over the last two decades have convincingly shown that the strong, electromagnetic, and weak forces are all closely related and are simply described by the "Standard Model." In particular the anticipated sixth quark, top, has been found at Fermilab, and the predicted properties of the $Z$ boson, one of the carriers of the weak force, have been tested to better than $0.1 \%$. Although there is now little doubt that the Standard Model is a very good description of the basic forces responsible for all atomic and nuclear physics, there remain many open questions. ${ }^{5}$

Perhaps the most urgent is to understand how masses of the elementary particles originate. To that end, new physics beyond what has been observed is required. The simplest possibility, the "Higgs Mechanism" predicts the existence of a fundamental Higgs Boson. Finding that elusive particle or whatever new physics is actually responsible for mass generation motivated the Superconducting Supercollider (SSC) and remains the primary goal of the next generation of colliders. A number of other interesting and more elaborate models have been proposed, but there is as yet no direct experimental evidence supporting any of them. Nevertheless, consistency of the Standard Model requires that the new physics responsible for mass generation

"Work supported by the Department of Energy, Contract NO. DE-AC02-98CH10886 


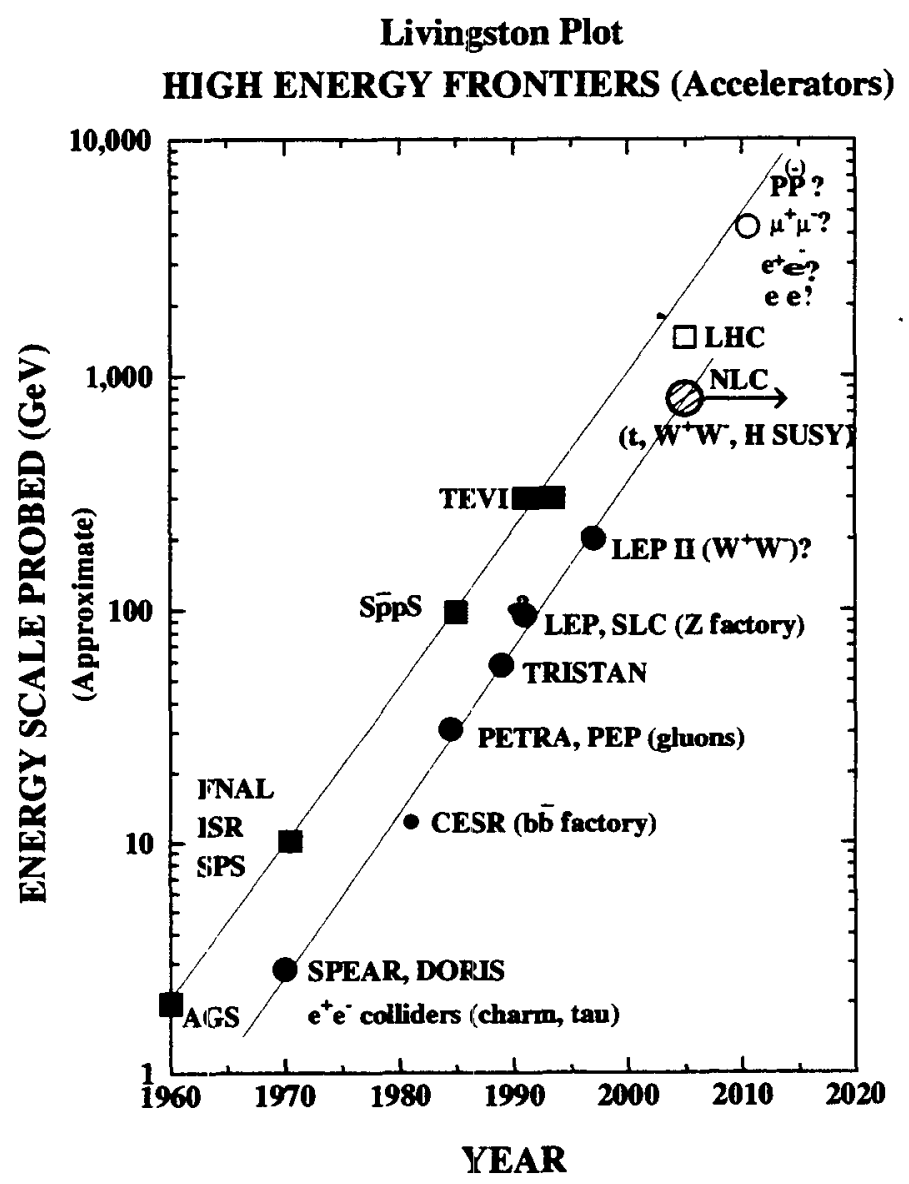

Fig. 1. Livingston Plot - Fligh Energy Frontiers (Accelerators) and their physics programs.

occur at an energy scale of less than about $1 \mathrm{TeV}$, i.e. within the range of the next generation of accelerators. In addition to the origin of mass, there are other compelling questions. For example, the observed matter-antimatter asymmetry in the universe is not understood. Also, astrophysical observations suggest that between $90 \%$ and $99 \%$ of the matter that makes up the universe is invisible. There are a number of possible candidates for this "dark matter," but none have been proven experimentally to exist.

Current operation of the Tevatron proton-antiproton collider at the Fermi National Accelerator Labora.tory and the LEP electron-positron collider at CERN are at the energy frontier of the field. The use of both proton-antiproton and electronpositron collisions is important in order to provide complementary information. At the energy frontier new particles never before observed are discovered and studied, providing unique insight into the laws of nature.

Table 1 illustrates (a summary of the standard model), minimal spectrum of 
Table 1. Elementary Particles and Their Properties

\begin{tabular}{lccccc}
\hline Symbol & Spin & Charge & Color & Mass $(\mathrm{GeV})$ & \\
\hline$\nu_{e}$ & $1 / 2$ & 0 & 0 & $<4.5 \times 10^{-9}$ & \\
$e$ & $1 / 2$ & -1 & 0 & $0.51 \times 10^{-3}$ & First \\
$u$ & $1 / 2$ & $2 / 3$ & 3 & $5 \times 10^{-3}$ & Generation \\
$d$ & $1 / 2$ & $-1 / 3$ & 3 & $9 \times 10^{-3}$ & \\
& & & & & \\
$\nu_{\mu}$ & $1 / 2$ & 0 & 0 & $<.16 \times 10^{-3}$ & \\
$\mu$ & $1 / 2$ & -1 & 0 & 0.106 & Second \\
$c$ & $1 / 2$ & $2 / 3$ & 3 & 1.35 & Generation \\
$s$ & $1 / 2$ & $-1 / 3$ & 3 & 0.175 & \\
& & & & & \\
$\nu_{\tau}$ & $1 / 2$ & 0 & 0 & $<2.4 \times 10^{-3}$ & Third \\
$\tau$ & $1 / 2$ & -1 & 0 & 1.777 & \\
$t$ & $1 / 2$ & $2 / 3$ & 3 & $174.3 \pm 5.1$ & Generation \\
$b$ & $1 / 2$ & $-1 / 3$ & 3 & 4.5 & \\
& & & & & \\
$\gamma$ & 1 & 0 & 0 & 0 & \\
$W^{ \pm}$ & 1 & \pm 1 & 0 & $80.39 \pm 0.04$ & \\
$Z$ & 1 & 0 & 0 & $91.187 \pm 0.002$ & \\
$g$ & 1 & 0 & 8 & 0 & \\
$H$ & 0 & 0 & 0 & $106<m_{H}<235(800)$ & \\
\hline
\end{tabular}

particles along with sorne of their basic properties. The fermions are grouped into three generations of spin 1/2 leptons and quarks which span an enormous mass range.

\section{Future Directions and Colliders}

High energy accelerators take us to new domains where top, Higgs, and "New Physics" can be directly produced and studied. The LHC, scheduled for 2005 will take us to $14 \mathrm{TeV}$ with very high luminosity $\simeq 10^{34} \mathrm{~cm}^{-2} \mathrm{~s}^{-1}$. Besides finding the Higgs, it will be capable of uncovering supersymmetry, $Z^{\prime}$ bosons, technicolor or many other scenarios writh "new physics" $\lesssim 1 \mathrm{TeV}$. Beyond those facilities, new ideas and technologies are required. The Next Linear Collider $\left(e^{+} e^{-}\right)$offers an exciting viable possibility. The Recent, growing enthusiasm for a $\mu^{+} \mu^{-}$collider with high energy $\gtrsim 3 \mathrm{TeV}$ and luminosity $>10^{35} \mathrm{~cm}^{-2} \mathrm{~s}^{-1}$, if feasible, would be a significant technological. leap forward.

High energy physicists are anxiously waiting for the next dramatic experimental discovery. Fortunately, anticipated future collider facilities offer broad discovery potential. The Fermilab main injector upgrade will allow the $p \bar{p}$ Tevatron to operate at $\sqrt{s} \lesssim 2 \mathrm{TeV}$ and luminosity $\sim 2 \times 10^{32}$. Those improvements broaden the discovery potential while allowing precision measurements and searches for rare $B$ and $\tau$ decays. The Higgs mass region of $110 \sim 130 \mathrm{GeV}$ may be explored via $W^{ \pm} H$ and $Z H$ associated production if the $H \rightarrow b \bar{b}$ mode is resolvable. ${ }^{4}$. Asymmetric 
$B$ factories provide new ways to explore CP violation. LEPII has achieved $e^{+} e^{-}$ center-of-mass energy of about $202 \mathrm{GeV}$ and will push its energy to $\sqrt{s} \simeq 204$ $\mathrm{GeV}$ or higher. If a stanclard model or SUSY Higgs with mass $\lesssim 110 \mathrm{GeV}$ exists, it should be found. Perhaps, they will also get a first glimpse of SUSY. Furthermore, the $W^{ \pm}$mass has been measured to $\leq \pm 45 \mathrm{MeV}$ at LEPII, and Fermilab, providing an interesting constraint on the Higgs mass via quantum loop relations.

In the longer term $(\sim 2005)$; the LHC $p p$ collider with $\sqrt{s}=14 \mathrm{TeV}$ should find the Higgs scalar or tell us it doesn't exist. If SUSY exists $\lesssim 1 \mathrm{TeV}$, it will be discovered. Hopefully, completely unexpected revelations will also be made.

Beyond the LHC, various collider options are possible. The Next Linear Collider (NLC) would start $e^{+} e^{-}$collisions at $\sqrt{s}=500 \mathrm{GeV}$ and be upgradeable to 1-1.5 $\mathrm{TeV}$. It would have high luminosity $>5 \times 10^{33}$ and polarization. The NLC also offers $\gamma \gamma, e^{-} e^{-}$, and $e^{-} \gamma$ collider options which expand its physics potential. The $e^{-} e^{-}$facility would feature high polarization in both beams and complement the overall discovery potential. There has beer. also some discussion of possible future $e^{+} e^{-}$colliders with $\sqrt{s} \geq 5 \mathrm{TeV}$, a major step, if achievable. The NLC will be a superb tool for studying the Higgs, SUSY, Technicolor etc. ${ }^{12,13}$

Other possibilities include a $\mu^{+} \mu^{-}$collider and Very Large Hadron Collider ( $p p$ with $\sqrt{s} \simeq 100 \mathrm{TeV}$ or more) which are less advanced. The muon collider concept is extremely interesting, but require series studies and technology demonstrations. An effort at BNL will aim to produce very intense muon beams and use them to do physics (such as $\mu^{-} N \rightarrow e^{-} N$ ). Such hands on efforts combined with a vigorous R\&D program could lead to the First Muon Collider (FMC). Various machine energies have been considered including $100 \mathrm{GeV}, 500 \mathrm{GeV}, 3 \mathrm{TeV}$ etc. The 3 $\mathrm{TeV}$ facility is complementary to the LHC. Recently, the concept of muon storage ring based Neutrino Source has generated considerable interest in the High Energy Physics community. Beside providing the first phase toward a muon collider, it would generate more intense and well collimated neutrino beams than currently available.

The Very Large Hadron Collider(VLHC) with $\sqrt{s} \simeq 100 \mathrm{TeV}$ and $\mathcal{L} \simeq 10^{35}$ looks technically feasible but is very expensive. Does a Very Large Hadron Collider with $\sqrt{s} \simeq 100 \mathrm{TeV}$ have viability? Our SSC experience suggests a prohibitive cost and difficult construction issues because of its size. However, new ideas about inexpensive magnets and tunnels and / or a new technology could offer hope for the needed significant recluction in cost. Figure 2 shows a schematic layout of the high energy accelerators to illustrate their relative sizes and energies. ${ }^{15}$

Figure 3 shows a schematic of a muon collider components.$^{15} \mathrm{~A}$ high intensity proton source is bunch compressed and focused on a heavy metal target. The pions generated are captured by a high field solenoid and transferred to solenoidal decay channel within a low frequency linac. The linac reduces, by phase rotation the momentum spread of the pions and of the muons into which they decay. Subsequently, the muons are cooled by a sequence of ionization cooling stages, and must be rapidly 


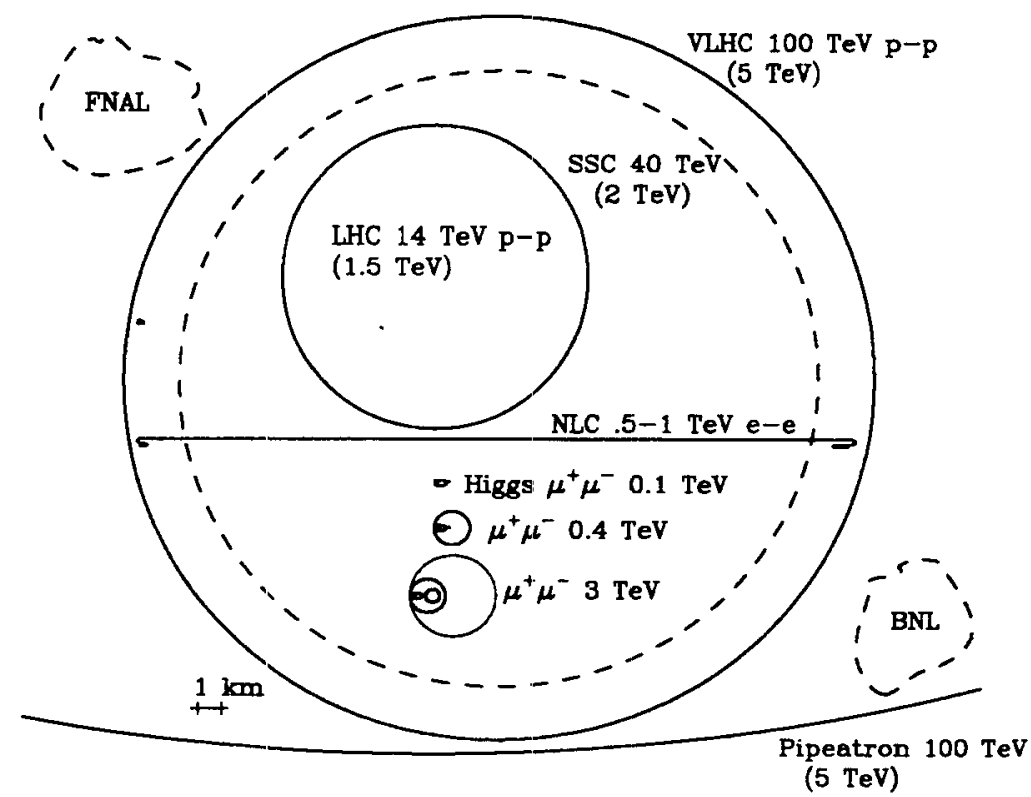

Fig. 2. Comparison of relative sizes of Muon Collider, Large Hadron Collider (LHC), and Next Linear Collider (NLC), relative to the BNL and FNAL sites.

accelerated to avoid decay. This can be done in recirculating accelerators (as at CEBAF) or in fast pulsed synchrotrons. Muon collisions occur in a separate high field collider storage ring with a single very low beta insertion. For more information on the Muon Collider and parameters under study, see references. ${ }^{15}$

\section{Laser plasma Acceleration}

The concept of Laser plasma acceleration is not new. It has for some time held the promise of producing intense high energy electron (and perhaps proton) beams. However, only with the recent development of compact terawatt laser systems could that concept be fully investigated in the laboratory. Among a number of laser accelerator concepts, laser wakefield accelerators have great potential for producing ultra-high field gradients of plasma waves excited by intense ultrashort laser pulses. The plasma structures have been considered as basic for future accelerators. The plasma has no breakdown limits since it is already ionized. Further, the plasma can support large longitudinal waves in which the electrons oscillate with $\omega_{p}=$ $\left(4 \pi e^{2} n_{0} / m\right)^{1 / 2}$, due to the space charge of the immobile ion background (regardless of the wavelength), $n_{0}$ is the plasma density in $\mathrm{cm}^{-3}$. Thus, in principle high intensity is possible.

One can create a relativistic plasma wave by properly phasing these oscillations such that $v_{\phi} \cong c$. So, the electron can reach relativistic energies before dephasing from the wave. The accelerating gradient of a relativistic plasma wave can be expressed as $n^{P u r t} \sqrt{n_{0}} V / \mathrm{cm}$, where $n^{P u r t}$ is the density of the perturbed wave. 


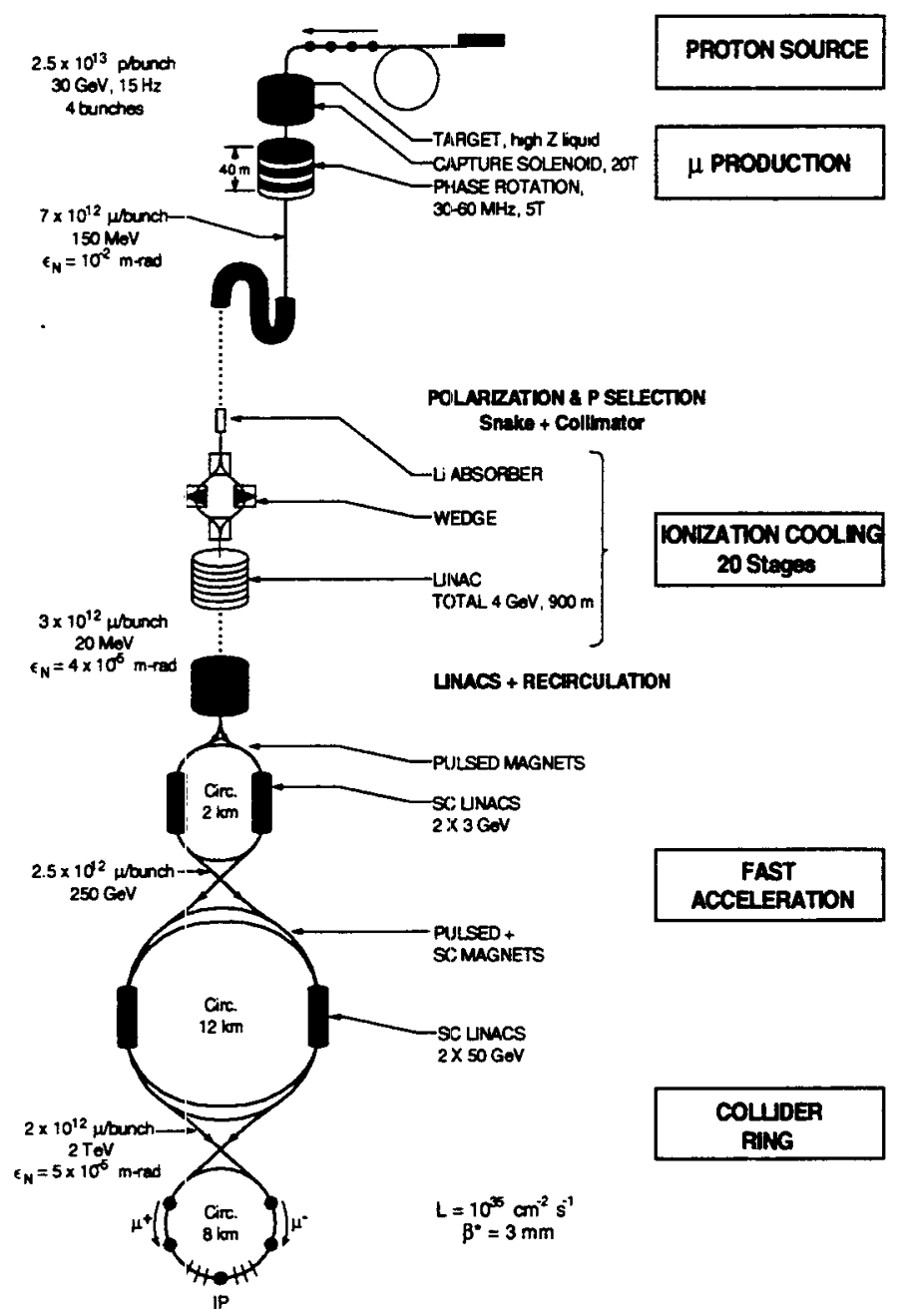

Fig. 3. Schematic of a $4 \mathrm{TeV}$ Muon Collider.

Relativistic plasma waves can be generated by propagating intense laser beams or intense particle beans. In the following sections we discuss laser driven acceleration schemes. The laser wake-field acceleration (LWFA), the plasma beat-wave accelerator (PBWA) and the self-modulated laser-wake-field accelerator (SMLWFA) concepts are illustrated in references. ${ }^{20}$

For illustration, consider a Gaussian laser pulse with peak power $\mathbf{P}$ and a temporal $e^{-1}$ half-width $\sigma_{z}$. Then, a peak amplitude of the accelerating wakefield may be expressed:

$$
E_{z}=\frac{\rho_{\text {igac }} P}{\sqrt{\pi} r m c^{2}} \frac{1}{L_{\text {Rayleigh }}} \frac{\lambda_{\text {laser }}}{\lambda_{\text {plasma }}} \frac{\pi \sigma_{z}}{\lambda_{\text {plasma }}} e^{-\left(\frac{\pi \sigma_{z}}{\lambda_{\text {plasma }}}\right)^{2}} .
$$

with the maximum amplitude occuring at $\lambda_{\text {plasma }}=\pi \sigma_{z}$. Here, $\rho_{v a c}$ is the vacuum 
resistivity, $L_{\text {Rayleigh }}\left(=\frac{\pi r_{\text {spot }}^{2}}{\lambda_{\text {laser }}}\right)$ is the vacuum Rayleigh length, and at the focus the spot radius is $r_{\text {spot }}$. $\lambda_{\text {laser }}$ is the laser wavelength, and $\lambda_{\text {plasma }}$ is the plasma wavelength.

For an acceleration length $L_{a c c}$ the masimum gain $(G)$ in energy $(E)$ for relativistic electrons is

$$
G E=e E_{z} L_{a c c}
$$

For resonant plasma density $n_{0}=\left(\pi r_{s p o t} \sigma_{z}^{2}\right)^{-1}$ with $L_{\text {Rayleigh }}, r_{e}$ as the clas-

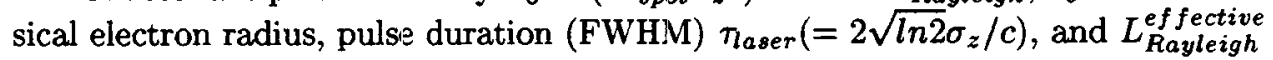
as the effective Rayleigh length for laser propagation in the plasma the maximum in energy may be estimated,

$$
G^{m a x} E[M e V] \simeq \frac{850 P[T W] \lambda_{\text {laser }}[\mu m]}{\tau_{\text {laser }}[\mathrm{fs}]} \times \frac{L_{\text {Rayleigh }}^{\text {effective }}}{L_{\text {Rayleigh }}}
$$

(for convenience, the units were inserted in the above formula).

Laser wakefield acceleration has the potential of producing an ultra high-field gradients of plasma waves. In a homogenous plasma, diffraction of the laser propagation limits the laser - plasma interaction distance to the vacuum Rayleigh length.

Although laser experiments have achieved successfully gradients of $100 \mathrm{GeV} / \mathrm{m}$, energies of $100 \mathrm{MeV}$ and accelerated nanocoulombs of charge using compact terawatt laser system ( $T^{3}$ lasers), most of the high gradient results are over very short (millimeter) distances. ${ }^{26}$

For practical application of the laser - driven accelerator concept, it is important to demonstrate high energy gain and high gradient acceleration for long distances. That is to achieve long interaction of an intense ultrashort laser pulse with under dense plasma. In the next section, parameters for a laser driven plasma - wake field accelerator which achieve the high energy requirements are also described. ${ }^{20}$

\section{High Energy Physics Requirements \& Laser Driven Accelerators}

There has been much theoretical and experimental work on the acceleration of particles using lasers, in the last two decades. Application of these new methods in high energy physics contirues to be of major interest and a motivating force, for the effort. The high energy physics requirements, can be summarized by the required collider operating "beam power" $\left(P_{\text {beam }}\right)$ "Luminosity" (L), and "Beamstrahlung energy spread" $\delta,:$

$$
\begin{gathered}
P_{\text {beam }}=2 \gamma m c^{2} N f=\eta P_{\text {wall }} \\
L=\frac{f N^{2}}{4 \pi \sigma_{x}^{2} R_{H / V}} \\
\delta=\frac{0.88 r_{e}^{3} N^{2} \gamma}{R_{H / V}^{2} \sigma_{x}^{2} \sigma_{z}}
\end{gathered}
$$

Where in the above formula, $\mathrm{N}$ is the number of particles per bunch, $\mathrm{f}$ is the frequency of the collider (bunch number times the repetition rate), $\sigma_{x}$ is the transverse 
rms bunch dimension at intersection, $R_{H / V}$ is the ratio of the horizontal to vertical beam size, $r_{e}$ is the classical electron radius, $\mathrm{E}$ the beam energy, $\sigma_{z}$ is the rms longitudinal bunch dimension. the efficiency $\eta$ is the ratio of the beam power to the wall-plug power. Economic considerations provide us with the wall-plug power $P_{\text {wall }}$, the high energy requirements provide $\gamma, \mathrm{L}$ and $\delta$, and the remaining unknown parameters $\mathrm{f}, R_{H / V}, \mathrm{~N}, \sigma_{x}, \sigma_{z}$, can be expressed in terms of the independent variables such that $\sigma_{z}$ scale approximately with the acceleration wavelength $\lambda_{\text {acc }}$. For convenience, the units are inserted in brackets in the following equations:

$$
\begin{gathered}
f[M H z]=0.05 \frac{L\left[10^{35}\right] E[T e V]}{\delta \sigma_{z}[\mathrm{~cm}] R_{H / V}} \\
\frac{N}{\sigma_{z}}\left[\mathrm{~cm}^{-1}\right]=6.4 x 10^{10}\left(\frac{\delta P_{\text {wall }}[\mathrm{GW}] R_{H / V}}{L\left[10^{35}\right] E^{2}[\mathrm{TeV}]}\right) \\
\sigma_{x}[n m] \approx 126 \sqrt{\delta \sigma_{z}[\mathrm{~cm}]} \frac{\eta P_{\text {wall }}[\mathrm{GW}]}{L\left[10^{35}\right] E^{3 / 2}[\mathrm{TeV}]}
\end{gathered}
$$

Applying the above requirements for the example of a $1 \mathrm{TeV}$ on $1 \mathrm{TeV}$ high energy linear collider to laser - driven accelerators, as can be seen from Table 2, puts very severe requirements on the accelerators. The two parameter sets, ${ }^{21}$ given in Table 2 are for examples of the new vacuum laser and laser plasma accelerators. ${ }^{20}$ The repetition rates must be very high and the emittance must be very small. The constraints are more severe, if one includes corrections (to the classical calculations) when calculating these parameters. Large quantum effects lead to additional problems. Changing the paraneters to reduce the quantum effects can lead to a regime where wake-fields become important.

The difficulties for the $1 \mathrm{TeV}$ application illustrates that more serious work is needed, before one can use the laser-driven accelerators for collider applications in the future.

It should be clear that, the real beam parameters such as Luminosity at the collision point in a linear accelerator, depends on various dynamic processes including beamstrahlung and disrujtion. For example, the (beam-beam or) beamstrahlung parameter $Y$ measures the ratio of the energy of a typical radiated photon (calculated classically) to the particle energy and can be expressed as $Y=\frac{\gamma B_{\text {collioson }}}{B_{\text {critical }}}$. Where $\gamma=E / m c^{2}, B_{\text {collision }}$ is the magnetic field at the collision point and $B_{\text {critical }}$ is the critical field. Beamstrahlung is in the classical limit if $Y<1$ and is in the strong quantum limit if $Y>1$. For linear collider designs $Y$ is usually kept less than 0.3 .

On the positive side, lasers are successfully used e.g., in photo - injectors and photon - photon $(\gamma \gamma)$ accelerators, etc. ${ }^{20}$ and will continue to be used in new laser experiments. For example, a new "forward ion acceleration in thin films driven by a high - intensity laser was achieved most recently where a collimated beam of fast protons (for the first time), with energies as high as $1.5 \mathrm{MeV}$ and total number of $\sim 10^{9}$, confined in a cone angle of $40 \pm 10$ degrees was observed. Acceleration field gradients $\sim 10 \mathrm{GeV} / \mathrm{cm}$ are inferred". 27 
Table 2. Example of Pararneters for Laser-Driven Accelerators Which Achieve the High Energy Requirements.

\begin{tabular}{|c|c|c|}
\hline $\begin{array}{l}\text { Parameters } \\
\text { Given }\end{array}$ & $\begin{array}{c}\text { Accelerator } \\
\text { Laser Plasma }\end{array}$ & $\begin{array}{c}\text { Accelerator } \\
\text { Vacuum Laser }\end{array}$ \\
\hline Energy $[\mathrm{TeV}]$ & 1 & 1 \\
\hline Luminosity $\left[\mathrm{cm}^{-2} \mathrm{~s}^{-1}\right]$ & $10^{35}$ & $10^{33}$ \\
\hline$\delta$ & .1 & .1 \\
\hline$\eta P_{w a l l}[G W]$ & .02 & .02 \\
\hline$\sigma_{z}[\mu m]$ & 10 & .1 \\
\hline$F_{H / V}$ & 100 & 100 \\
\hline$\lambda_{\text {accel }}[\mu m]$ & 100 & 1. \\
\hline$E_{z}[\mathrm{GeV} / \mathrm{m}]$ & 10 & 1 \\
\hline$\beta^{*}=\sigma_{z}[\mu \mathrm{m}]$ & 10 & .1 \\
\hline$l / a$ & - & 10 \\
\hline Parameters & A.ccelerator & Accelerator \\
\hline Calculated for & Laser Plasma & Vacuum Laser \\
\hline$N / \sigma_{.:}\left[\mathrm{cm}^{-1}\right]$ & $1.3 \times 10^{10}$ & $1.3 \times 10^{10}$ \\
\hline$N$ & $1.3 \times 10^{7}$ & $1.3 \times 10^{5}$ \\
\hline$\sigma_{2}[\mathrm{~cm}]$ & $2.5 \times 10^{-9}$ & $2.5 \times 10^{-10}$ \\
\hline$f[M H z]$ & 4.9 & 490 \\
\hline$\tau_{b}[\mu m]$ & $2.3 \times 10^{-} 3$ & - \\
\hline$r_{b} \omega_{p} / c$ & $10^{-4}$ & - \\
\hline$n_{p}\left[\mathrm{~cm}^{-3}\right]$ & $1.1 \times 10^{17}$ & - \\
\hline$\delta n_{p} / n_{p}$ & .1 & - \\
\hline$\Delta E[M e V]$ & - & $4.8 \times 10^{-3}$ \\
\hline$\varepsilon_{N}=\gamma \sigma_{x}^{2} / \beta^{*}[m]$ & $11.3 \times 10^{-10}$ & $1.3 \times 10^{-10}$ \\
\hline
\end{tabular}

\section{Discussion}

Laser driven accelerators are very promising. They offer the possibility of producing extremely high accelerating gradients greater than $100 \mathrm{GeV} / \mathrm{m}$. As such, they could lead to compact future generations of $e^{+} e^{-}$and $e^{-} e^{-}$colliders with energies well beyond the $\mathrm{TeV}$ scale. Also those facilities should be capable of preserving polarization, an important feature. In the short term it would be useful to pursue a "stageable" concept that promises e.g., $1 \mathrm{GeV} / \mathrm{m}$, instead of few $\mathrm{GeV}$ (or $>100$ ) from a few Rayleigh lengths, if they are to be simply used as (or part of) future colliders.

The present generation of high energy colliders are based on RF acceleration where the acceleration is limited to electrical break down from material surfaces. Ultra high energy colliders $\sim 10 \mathrm{TeV}$ or more if based on conventional technology would lead to prohibitively large scales both in size and cost. Before such energies can be pursued, new technology must be introduced. However, to use the laser driven accelerators for practical application in the future more work is needed.

High energy accelerators take us to new physics domains. Such facilities, if feasible, would represent a significant technological leap forward. These could be 
new methods of acceleration or the use of new technologies, for example lasers in electron-electron linear colliders, or new methods of experimentation such as introduction of plasma at intersection point (compensated $e^{+} e^{-}$collisions). These ideas and technologies should be considered to the degree they will bring revolutionary, not evolutionary changes to accelerators and to the way collisions at ultra high energies can be achieved. For more details see references. ${ }^{1-27}$

\section{References}

1. See [2], [3] and references therein.

2. Z. Parsa (Editor), Future High Energy Colliders AIP-Press CP 397 (1997).

3. Z. Parsa, Collision Crossroads, CERN Courier, International Journal of High Energy Physics, Vol. 37, No. 2. March 1997, Ed. G. Fraser; Z. Parsa, New Ideas for Particle Áccelerators, ICFA Beam Dynamics News Letter, No. 14,(Âug. 1997) Eds. K. Hirata, J.M. Jewett.; ibid, Lasers and Future High Energy Colliders, STS-Press, 823 (1997).

4. See e.g. [5] - [11]

5. W.J. Marciano, Keynote Address in Proc. of Snowmass 1996 and Proc. of Santa Barbara symposium on "Future High Energy Colliders" 1996 Ed. Z. Parsa, AIP CP 397, pp 11-25 (1997).

6. C-N. Yang, Oskar Klein Memorial Lecture; Phys. Today 33,42 (1980); A. Zee, "Fearful Symmetry", Macmillan 1986.

7. S. Weinberg, Phys. Rev. Lett. 19 (1967) 1264; A. Salam, in Elementary Particle Theory, ed N. Svartholm (Almquist \& Wiksells, 1968) p. $36 \overline{7}$.

8. See A. Sirlin, Comments on Nucl. and Part. Phys., 21, 287 (1994).

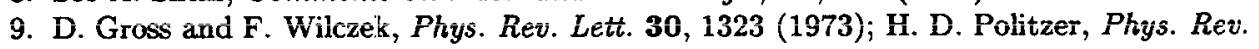
Lett. 30, 1346 (1973).

10. W. Marciano, Phys. Rev. D29, 580 (1984).

11. A. Stange, W. Marciano, and S. Willenbrock, Phys. Rev. D50, 4491 (1994).

12. S. Kuhlman et al., Phyisics Goals of the Next Linear Collider, BNL report 63158.

13. ZDR Report for NLC, SLAC, May 1996 and updates (1999).

14. V. Telnov, in Future High Energy Colliders Ed. Z. Parsa, AIP CP 397 (1997) and in New Methods of Particle Accelerations Techniques and Sources, Ed. Z. Parsa, AIIP C̄P 396 (1997); J. Irvine, Private Comm.

15. See e.g. [15] - [19]; J. Norem and K. McDonald private comm.: provided files (drawings) for Fig. 3 and Fig. 2, respectively.

16. C.M. Ankenbrandt etal, Status of mwon collider research and development and future plans, Phys. Rev. ST Accel. Beams 2, 081001 (1999), and references therein.

17. V. Barger, M. Berger, K. Fujii, J. Gunion, T. Han, C. Heusch, W. Hong, S. Oh, Z. Parsa, S. Rajpoot, R. Thun, W. Willis, BNL-61593 (1995).

18. Z. Parsa, New High Iniensity Muon sources and Flavor Changing Neutral Currents, World scientific Publishing, pp 147-153 (1998).

19. Z. Parsa, Polarization and Luminosity requirements for the First Muon Collider, in AIP Conf. Proc. 472, pp. 251-259, (1998).

20. See e.g., [20] - [25]

21. J. Wurtle, A. Sessler, et al. DFP/DPB Summer Study, New Directions for HEP, Snowmass, CO, June 25 - July 12 (1996); Also see e.g., Advanced Accelerator Concepts, Ed. W. Lawson, C. Bellamy, D. Brosius, AIP Conf. Proc. 472 (1998); e.g., see T. Katsouleas p19; S. Chattopadhyay, P. Chen pp.169-171.

22. Z. Parsa, (Editor) "New Methods of Particle Accelerations Techniques and Sources", AIP CP 396 (1997); also papers and references therein. 
23. Z. Parsa, Lasers and New Methods of Acceleration, STS-Press, pp 823-830 (1997), and papers therein: e.g. K. Nagajima p 778; ibid et al., Phys. Rev. Lett. , 74, 4428 (1995); T. Tajima and J. M. Dawson, Phys. Rev. Lett. 43, 267 (1979);D. Umstadter et al., Science273, 472 (1996).

24. Z. Parsa, Development of the Alternate Entry Port for the ATF, 0-7803-1203-1/93 IEEE (1993); ibid, Analysis of a High Brightness Photo Electron Source with Self-field and Wake-field Effects, NIM A318 (1992) 259-264, North Holland.

25. Z. Parsa, New Modes of Particle Accelerations, Laser and Particle Beam Journal, Vol. 13, No. 5 (1997); Cambridge Univ. Press, 0263-0346/97. Also see updates, e.g. M. Perry and other Laser presentations in the proceedings of e-e-g9, Santa Cruz, December 1999.

26. P. Mont et al., Phys. Rev. Lett. 74, 2953; (1995); E.S. Sarachic, G. Shappert, Phys. Rev. D1, 2738 (1970); G. Malk et al., Phys. Rev. Lett. 79, 2053 (1997).

27. D. Umstadter et al., private comm.,Phys. Rev. Lett. (2000). 